Article

\title{
A Telemetry, Tracking, and Command Antennas System for Small-Satellite Applications
}

\author{
Paolo Squadrito ${ }^{1, *(\mathbb{D}}$, Patrizia Livreri ${ }^{2}\left(\mathbb{D}\right.$, Loreto Di Donato $^{3}\left(\mathbb{D}\right.$, Concetto Squadrito $^{4}(\mathbb{C}$ \\ and Gino Sorbello 3 (DD \\ 1 Antennas, Propagation and Millimeter-Wave Systems section at the Department of Electronic Systems, \\ Aalborg University, 9220 Aalborg, Denmark \\ 2 Department of Engineering, University of Palermo, Viale delle Scienze Ed.9, 90128 Palermo, Italy; \\ patrizia.livreri@unipa.it \\ 3 Department of Electrical, Electronics and Computer Engineering, University of Catania, viale A. Doria 6, \\ 95126 Catania, Italy and and Consorzio Nazionale Interuniversitario per le Telecomunicazioni (CNIT), Viale \\ G.P. Usberti, I-43124 Parma, Italy; loreto.didonato@dieei.unict.it (L.D.D.); gino.sorbello@unict.it (G.S.) \\ 4 Sicilsat Communications s.r.l, Via della Resistenza 44, 95030 Pedara, Italy; c.squadrito@sicilsat.com \\ * Correspondence: pas@es.aau.dk; Tel.: +45-9940-8727
}

Received: 15 May 2019; Accepted: 14 June 2019; Published: 18 June 2019

\begin{abstract}
Circularly polarized (CP) antennas are used in space applications for telemetry tracking and command (TT\&C). In this paper, we present an antenna for a TT\&C system that can employ only four antennas to achieve a wide quasi-isotropic coverage. The proposed single-feed circular polarized annular patch antenna is low profile and compact. It exhibits a wide impedance bandwidth of $19.7 \%$, wide-angle circular polarization with an on-axis maximum gain higher than $6 \mathrm{dBi}$ and an axial ratio below $4 \mathrm{~dB}$ in the frequency band 2.0-2.3 GHz. The developed low-cost antenna can be used for small-satellite data downlink where only two right hand circular polarized (RHCP) antennas and two left hand circular polarized (LHCP) antennas would be required since each antenna has an adequate CP gain on a wide angle. A prototype antenna has been fabricated and measured. The experimental validation shows a good agreement of the measured impedance bandwidth, axial ratio, and far-field pattern with the ones predicted by numerical full-wave study.
\end{abstract}

Keywords: circular patch; circular polarization; TT\&C; small satellites

\section{Introduction}

The number of space missions involving small satellites in their different sizes is exponentially increasing. The reasons for this growth are mainly related to the low cost and the flexibility that a small-satellite-oriented approach can offer. Indeed, several kinds of missions, such as communication [1], remote sensing [2] and Earth observation [3], are now possible despite the small dimension of the platform. In general, multiple nanosatellites, designed for different applications, can be placed in the same orbit or different orbits by the same launcher. The TT\&C (Telemetry Tracking and Command) link of a satellite is of prime importance, right from the time of its launch to the end of the mission. These subsystems provide an uplink for command signal, a downlink for monitoring signal, and various health parameters through telemetry and tracking information for monitoring its position in orbit. Right after the launch, a satellite is affected by unstable attitude conditions, therefore it must be stabilized before the payload became operational. TT\&C antennas are a key component in this phase to provide the attitude signal and the position to the ground station and receive the data needed for the de-tumbling operations. The S-band in the frequency interval 2.0-2.3 GHz is used for TT\&C [4]. The specification for a TT\&C antenna requires full spherical coverage, since the orientation of the satellite is uncertain, and circular polarization due to the Faraday effect in the atmosphere. 
It is well known that circular polarization (CP) can be obtained from linear orthogonal polarizations with an appropriate feed network to radiate them in-phase quadrature [5-9]. The above method has many advantages such as wide bandwidth and dual sense of polarization when a quadrature hybrid is used in the feed network [5]. However, the feeding structure complicates antenna design and fabrication. In [7] an S-band TT\&C antenna employing bow-tie dipoles excited by an appropriate feeding network has been presented. To avoid complicated feeding networks, $\mathrm{CP}$ can also be generated with perturbation technologies [10-14]. This approach results in compact single-feed antennas, but such design generally has very limited bandwidth. The reduced bandwidth, typically a few percent, does not represent a limitation for relatively narrowband short-range communication systems $[12,15]$. A CP capacitively coupled single-feed high-gain patch antenna for wireless power transfer has been presented in [16]. However, the 3-dB axial ratio (AR) bandwidth is only $2 \%$.

In this paper, we present a wide-bandwidth TT\&C antenna system employing multiple S-band annular ring antenna in a stacked configuration [17] with over $19.7 \%$ of bandwidth achieved thanks to a double resonance. Circular polarization is obtained in the proposed single fed antenna by exciting two orthogonal quasi-symmetrical polarizations. A parametric study is presented and a prototype, adopting space-qualified materials, is realized. Measured scattering parameters, AR, and antenna gain agree well with simulation results.

\section{Antennas System}

\subsection{Configuration}

Two RHCP antennas and two LHCP antennas are used for the proposed TT\&C system. These antennas have been fabricated and have been installed at the edge of the satellite's payload (a $70 \mathrm{~cm}$ diameter $\mathrm{X}$-band dish antenna) to avoid shadowing from the payload itself. The position of the TT\&C antennas is shown in Figure 1.

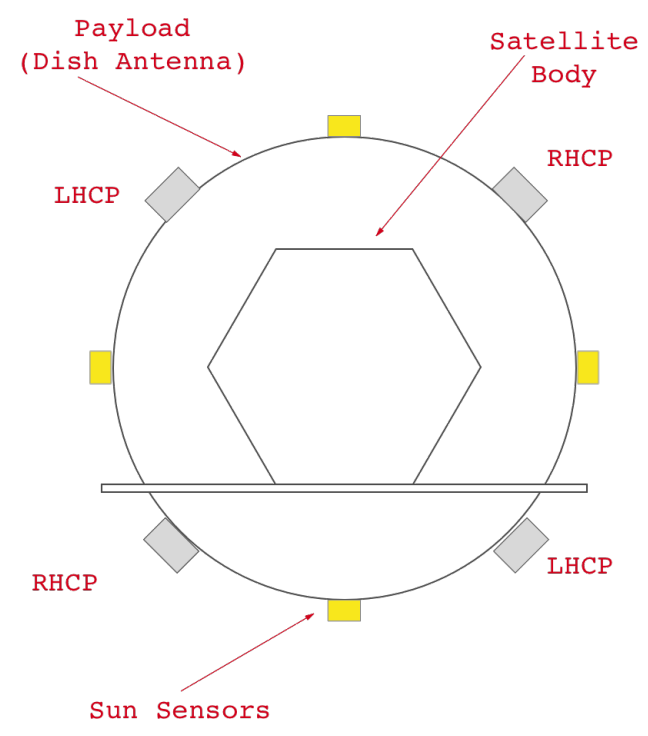

Figure 1. Top view of the satellite. The four antennas (in grey) are placed on the satellite's payload (a dish antenna) along with the sun sensor (in yellow).

Each antenna covers a hemispheric region; therefore, the antennas altogether provide a quasi-isotropic radiation pattern. In this way, during tumbling, all antennas are used to provide TT\&C communications with the ground station.

The goal of the study was to obtain a wideband $\mathrm{CP}$ antenna with an axial ratio below $4 \mathrm{~dB}$ from 2.0 GHz to 2.3 GHz. Moreover, for the CP gain an 80-deg half-power beamwidth (HPBW) is required 
i.e., provided that the maximum $\mathrm{CP}$ gain is reached in boresight direction (at $\theta=0$ ) we require: $G(\theta) / G_{\max }>1 / 2$ for $\theta \in[-40,40]$ deg.

To achieve wide $4 \mathrm{~dB}$ axial ratio bandwidth multiple resonances are necessary; moreover, to easily control CP over a wide frequency bandwidth, the possibility to independently tune the amplitude and phase of the two linearly polarized components of the radiated electromagnetic field at different frequencies is desirable.

\subsection{Antenna Element}

The layout of the proposed antenna element is shown in Figure 2.

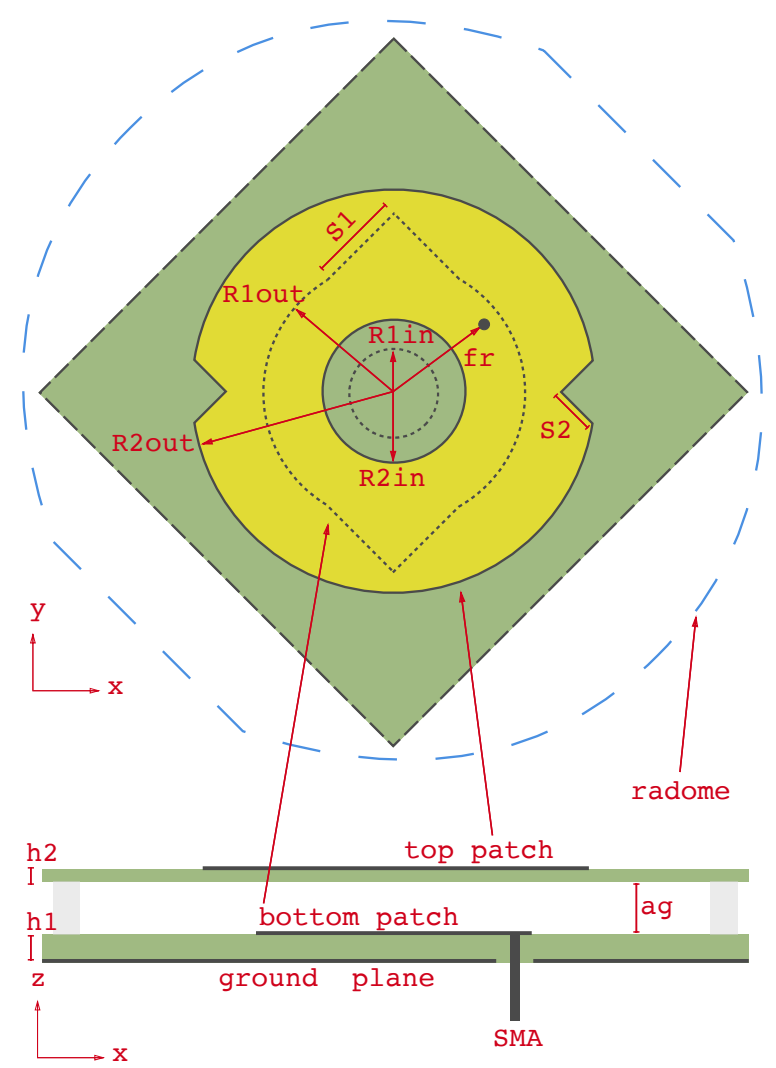

Figure 2. Top view and side view of the proposed antenna layout. The metallizations are represented in yellow, the substrates in green, and the radome in dashed blue. Lines with the same dash step belong to the same layer.

In this design, two annular patch antennas are employed in a stacked design. In the bottom layer, the first annular patch antenna and the ground plane are printed on a Rogers TMM4 with a dielectric constant of $\epsilon_{r 1}=4.50$ (simulated $\epsilon_{r 1}=4.661$ ) and with a loss tangent of $\tan \delta=0.002$. This metallization is represented in Figure 2 in dashed line with inner and outer radius $R 1_{\text {in }}$ and $R 1_{\text {out }}$ respectively.

Due to the presence of triangular extensions (with side S1) the $\mathrm{TM}_{11}$ mode, of the unperturbed annular patch, splits into two near degenerated orthogonal modes: mode 1 and mode 2 as shown in Figure 3. The equivalent resonant length along $x$ of mode 1 is larger than the equivalent length along $y$ of mode 2 . The feed point is at coordinate $\left(f_{x}, f_{y}\right)=\left(f_{r} \cos \phi, f_{r} \sin \phi\right)$ and, being $\phi=45 \mathrm{deg}$, it well couples both modes. 


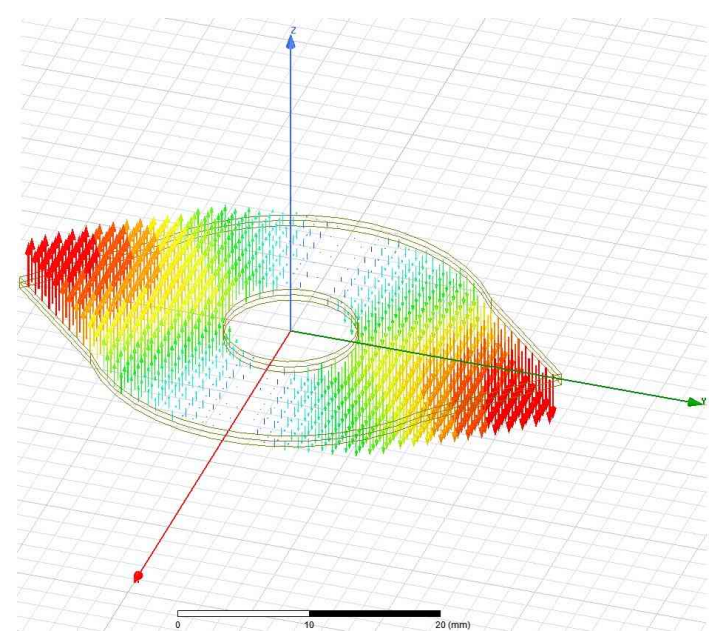

(a)

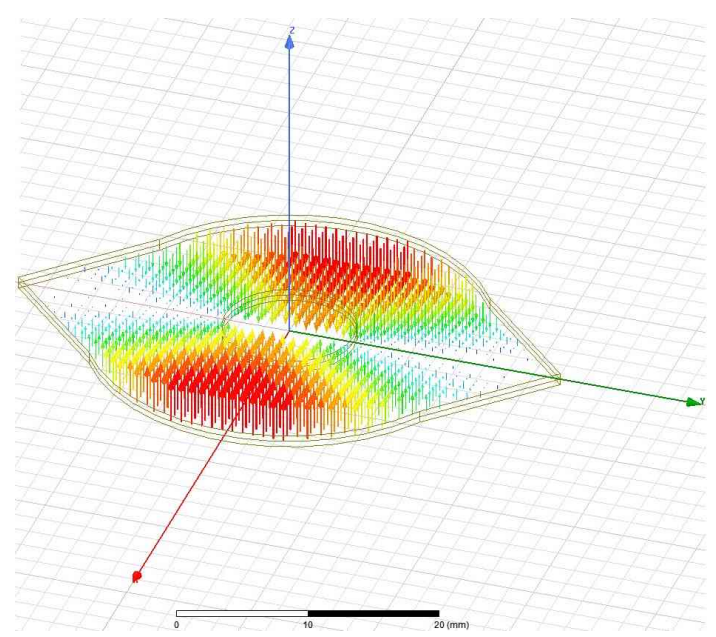

(b)

Figure 3. First two lower order mode supported by an ideal cavity model (with magnetic wall on the patch perimeter). The $\mathrm{TM}_{11}$ mode splits into two near degenerated orthogonal modes: mode 1 and mode 2. (a) Mode 1 lower frequency; (b) Mode 2 higher frequency.

The two modes naturally oscillate in-phase quadrature for an appropriate choice of the geometric parameters in, particular, we will show that the phase difference in the radiated field can be controlled modifying the equivalent length along $x$ and $y$ acting for example on $S 1$. A second coupled patch is printed on a Rogers RO4003 (Rogers Corporation, Chandler, AZ, USA) with a dielectric constant of $\epsilon_{r}=$ 3.607 and with a loss tangent of $\tan \delta=0.002$ and suspended above the main patch. These materials have been chosen to comply with the mechanical and thermal requirement. The inner and outer radii of the top annular patch are $R 2_{\text {in }}$ and $R 2_{\text {out }}$ respectively. An air gap is obtained between the two layers by using four cylindrical spacers made of PEEK and four screws made of Delrin POM. In this case, the cavity under the patch is filled with both Rogers RO4003 and air. The top patch geometric dimensions are sensibly larger than bottom ones, $R 1_{\text {in }}$ and $R 1_{\text {out }}$, since the equivalent dielectric constant filling the patch cavity is a value in between 1 (air) and 3.27 (Rogers RO4003). Also for the top patch cavity, the equivalent resonant length along $x$ is larger than the one along $y$ since a pair of triangular shaped aperture (with side S2), are etched (subtracted) along the $y$ direction and also in this case for an appropriate choice of the design parameters CP can be optimized. By coupling the patch in the bottom layer with the one in the top layer a wider operational bandwidth is achieved. Moreover, thanks to the multiple resonances and the several tuning geometric parameters (top and bottom inner and outer radius as well as the extension/slit) CP can be optimized at the same time for slightly different frequencies, in the band of interest, for wideband operation. Table 1 shows the dimensions of the proposed antenna. If the antenna is mirrored i.e., if the feed point is placed along the opposite diagonal at $\left(f_{x}, f_{y}\right)=\left(f_{r} \cos \phi,-f_{r} \sin \phi\right)$ the polarization sense can be switched from LHCP to RHCP.

Table 1. Antenna geometric parameters (mm). 1 refers to bottom patch, 2 refers to the top patch.

\begin{tabular}{cccccccccccc}
\hline R2out & R2in & R1out & R1in & S1 & S2 & fr & fx & fy & h2 & h1 & ag \\
\hline 23.837 & 8.351 & 16.061 & 5.261 & 12.204 & 5.76 & 14.6 & 10.323 & 10.323 & 1.524 & 3.175 & 11.17 \\
\hline
\end{tabular}

Values reported in Table 1 are the final set of parameters used for the manufactured antenna. The above geometric values have been fixed after a parametric study carried out on several antenna parameters by standard finite-element-method full-wave simulations considering the frequency band of interest as well as the angular mask for the radiated field. Circular polarization is obtained by controlling the cavity parameters in order to have the radiated field with $\theta$ and $\phi$ component of equal amplitude and in-phase quadrature. 


\section{Parametric Study}

The complex number:

$$
p=\mathrm{j} \frac{E_{\theta}}{E_{\phi}}=\mathrm{j} \frac{a_{\theta}}{a_{\phi}} \mathrm{e}^{\mathrm{j}\left(\psi_{\theta}-\psi_{\phi}\right)}
$$

where

$$
E_{\theta}=a_{\theta} \mathrm{e}^{\mathrm{j} \psi_{\theta}} \quad \text { and } \quad E_{\phi}=a_{\phi} \mathrm{e}^{\mathrm{j} \psi_{\phi}} .
$$

is referred as the linear polarization ratio [18] and can be used to evaluate the polarization state. In particular we have CP for $p= \pm 1$ (i.e., for $a_{\theta}=a_{\phi}$ and $\psi_{\theta}-\psi_{\phi}= \pm \pi / 2 \mathrm{rad}= \pm 90 \mathrm{deg}$ ). In the remaining of the paper we adopt the following shorthand notation $\left|E_{\theta}\right|=a_{\theta}$ for the modulus of the electric field $\theta$-component; $\left|E_{\phi}\right|=a_{\phi}$ for the modulus of the electric field $\phi$-component; $\angle E_{\theta}=\psi_{\theta}$ for the phase of the electric field $\theta$-component and $\angle E_{\phi}=\psi_{\phi}$ for the phase of the electric field $\phi$-component. When the ratio $\left|E_{\theta}\right| /\left|E_{\phi}\right|$ is equal to one and the phase the phase difference $\angle E_{\theta}-\angle E_{\phi}$ is $90 \mathrm{deg}$ we are in presence of circular polarization. These two quantities are more directly linked to the geometric parameters used in the optimization and will be used below to discuss some results of the parametric study where appropriate.

\subsection{Triangular Extensions and Slits}

The triangular extensions $S 1$ of the bottom patch and the triangular slits $S 2$ of the top patch, by loading only one of the two modes of the corresponding patch cavity, modify only one of the resonant length of the two degenerate modes with respect to the other. This can be used to effectively control the phase difference of the radiated field that is related to the unequal resonant lengths.

As shown in Figure 4 this allows fine control of phase difference of the linearly polarized components of the radiated field; on the contrary the ratio $\left|E_{\theta}\right| /\left|E_{\phi}\right|$ in less affected. From Figure 4e it is apparent that the effect of $S 1$ on the phase difference is slightly larger at lower frequencies in relation to the fact that the bottom patch has a lower resonant frequency.

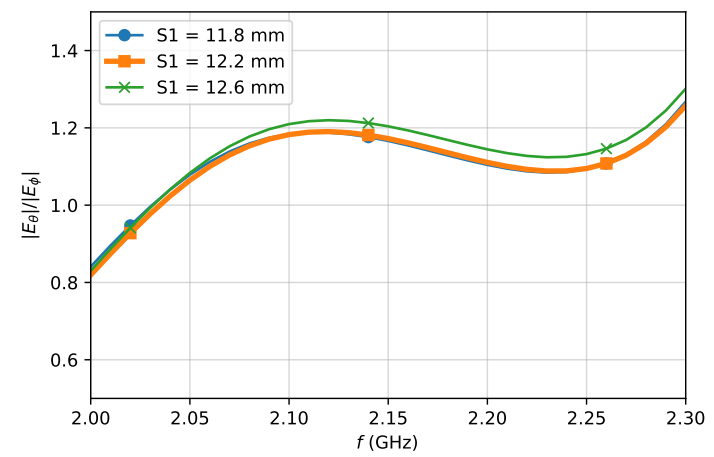

(a)

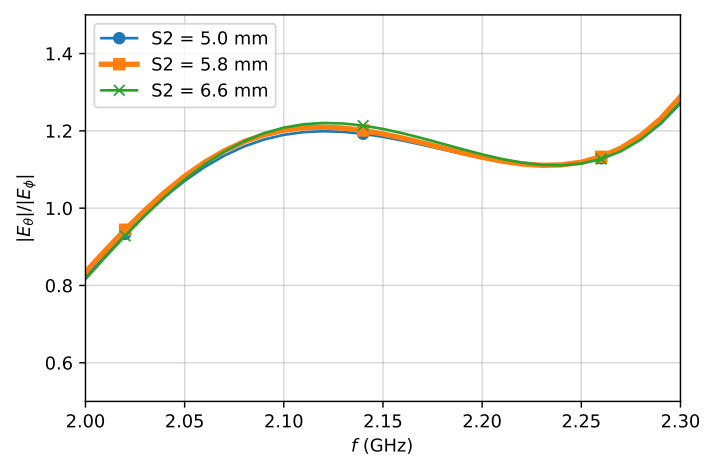

(b)

Figure 4. Cont. 


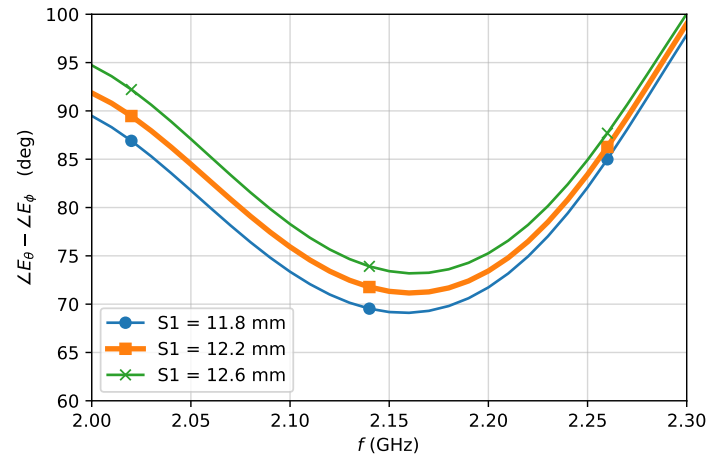

(c)

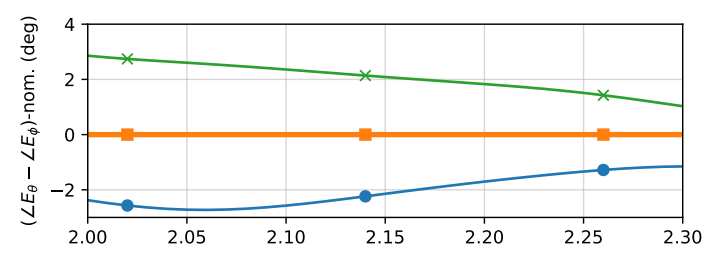

(e)

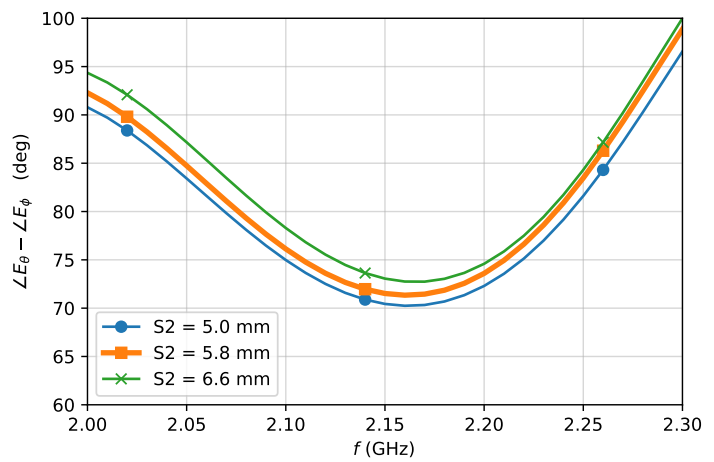

(d)

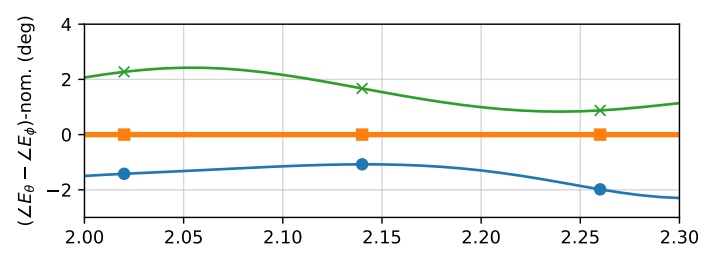

(f)

Figure 4. Effect of the lug $S 1$ variation and slit $S 2$ variation; all other parameters including the radome are fixed to nominal ones. (a) Ratio $\left|E_{\theta} / E_{\phi}\right|_{\theta=0}$ versus $S 1$; (b) Ratio $\left|E_{\theta} / E_{\phi}\right|_{\theta=0}$ versus $S 2$; (c) Phase difference $\angle E_{\theta}-\angle E_{\phi}$ versus $S 1$; (d) Phase difference $\angle E_{\theta}-\angle E_{\phi}$ versus $S 2$; (e) Phase difference minus "phase difference at nominal $S 1$ " vs. $S 1$; (f) Phase difference minus "phase difference at nominal $S 2$ " vs. $S 2$.

\subsection{Air Gap}

Many parameters have been considered and tuned during the optimization process. Among these, the air gap can be easily tuned also in the last stages of the fabrication (see Figure 5). The air gap directly modifies the upper cavity height. This has an important loading effect and an influence on the effective resonant lengths along $x$ and $y$ of the top patch cavity (and to a lesser extent also on the effective resonant lengths of the bottom one).

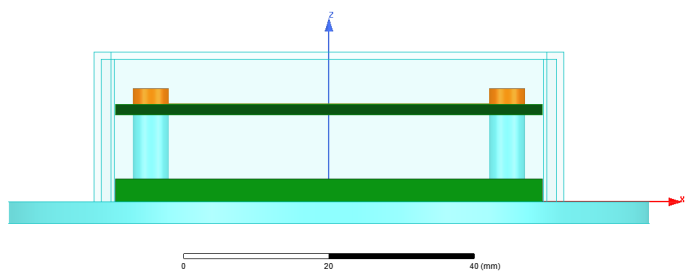

(a)

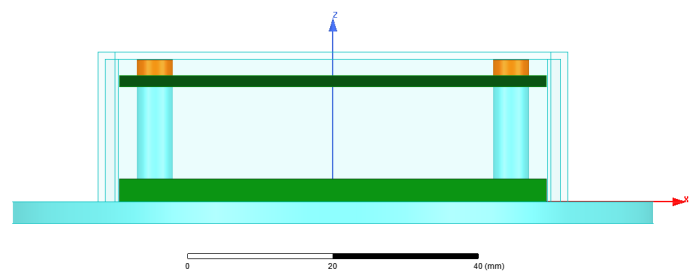

(b)

Figure 5. Side view of the proposed antenna showing the air gap variation from $9 \mathrm{~mm}$ (a) to $13 \mathrm{~mm}(\mathbf{b})$; all other parameters including the radome are fixed to nominal ones.

From Figures 6 and 7, where we show the co- and cross-polar gain at the central frequency of $2.15 \mathrm{GHz}$ for different spacing between the top and bottom substrates, it is apparent that the air gap has a great influence on the cross-polar level, this is due to the fact that the spacing affects the ratio $\left|E_{\theta}\right| /\left|E_{\phi}\right|$ between the two linear polarization. 


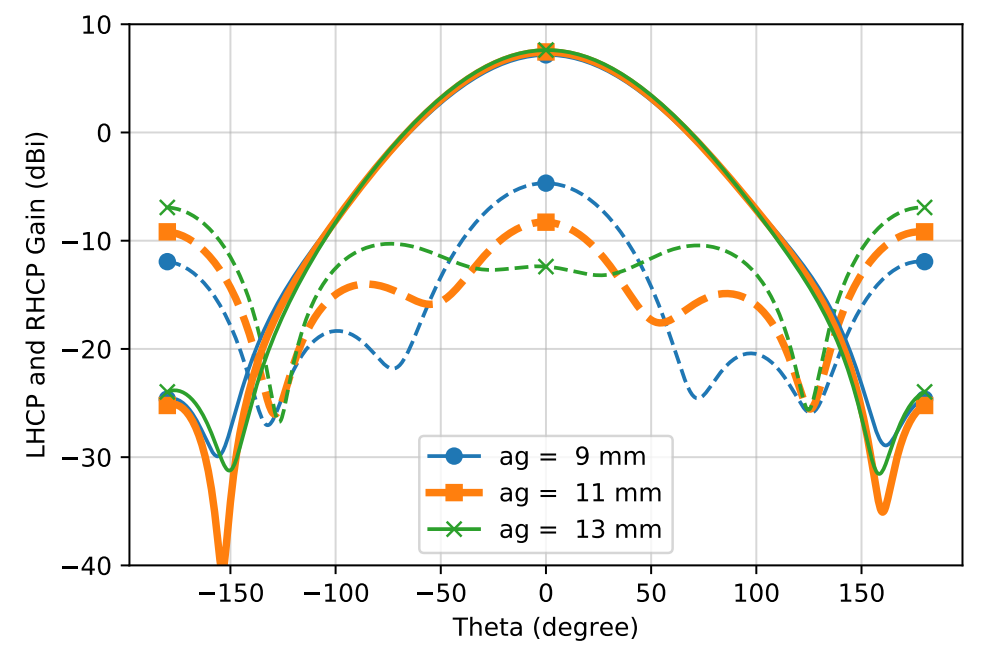

Figure 6. Co-and cross-polarization gain as function of the air gap at $f=2.15 \mathrm{GHz}$ and cut plane $\phi=0$ deg. Co-polar RHCP gain solid line and cross-polar LHCP gain dashed line curves.

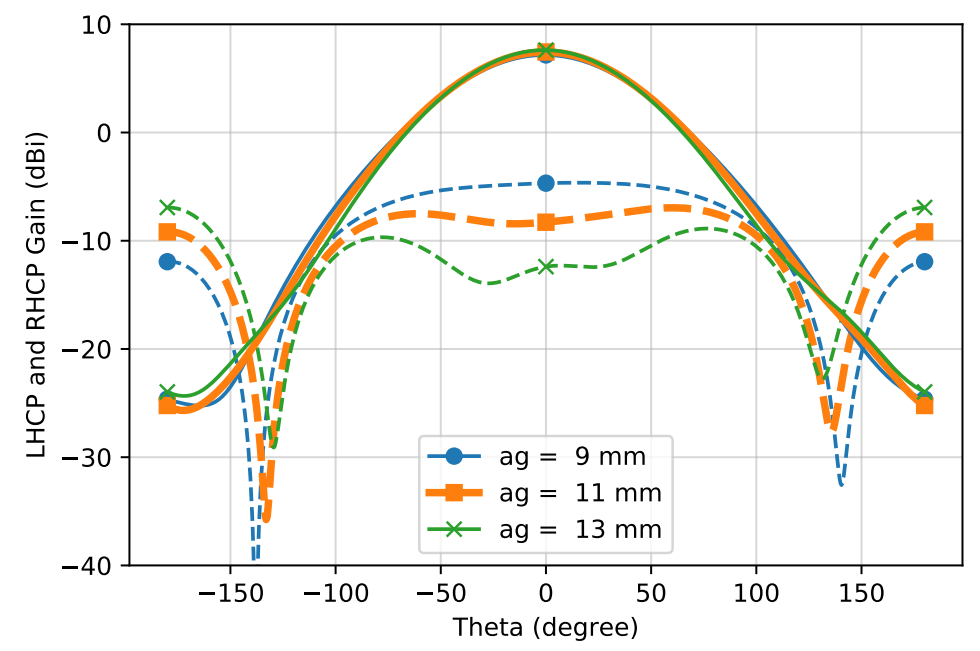

Figure 7. Co-and cross-polarization gain as function of the air gap at $f=2.15 \mathrm{GHz}$ and cut plane $\phi=90$ deg. Copolar RHCP gain solid line and cross-polar LHCP gain dashed line curves.

From the same figures, it is less evident that the air gap has also a secondary effect on the antenna co-polar gain that according to the theory, has an increase of $\approx 0.4 \mathrm{~dB}$ when the gap is increased from $9 \mathrm{~mm}$ to $13 \mathrm{~mm}$. The impact of the air gap on the quality of $\mathrm{CP}$ is more evident from axial ratio plotted for different value of the air gap as shown in Figure 8. From the parametric axial ratio plots, it is apparent that $a g$ has a great impact on the second resonance at higher a frequency.

The influence of the air gap on the $\mathrm{CP}$ can be better understood if we observe the ratio between the linearly polarized component $E_{\theta}$ and $E_{\phi}$ of the radiated field. In Figure 9 we show the $E_{\theta}$ to $E_{\phi}$ ratio for the cut $\phi=0 \mathrm{deg}$. By varying the air gap, the ratio between $\left|E_{\theta}\right|$ and $\left|E_{\phi}\right|$ far-field components can be controlled and tuned to the target unit value. 


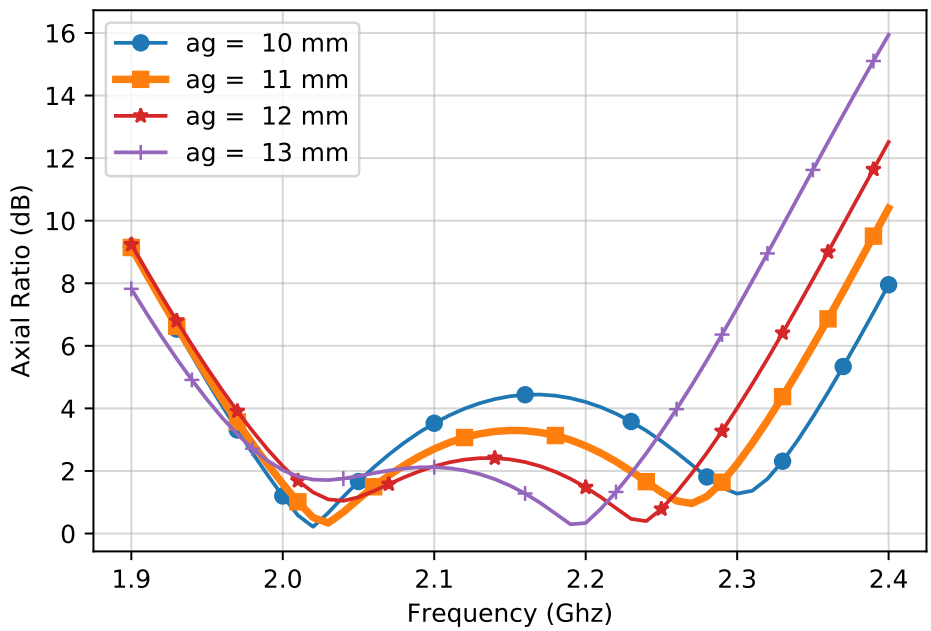

Figure 8. Axial ratio as function of the air gap. The presence of the radome is included in this study. $\phi=45 \mathrm{deg}$. The actual air gap of the realized antenna is $11.17 \mathrm{~mm}$. Variations in this parametric study are 10,11, and $12 \mathrm{~mm}$. The effect of the air gap variations is more important at higher frequencies.

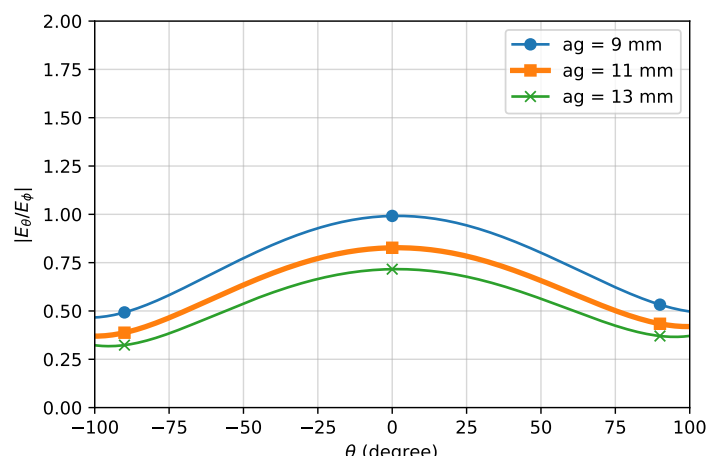

(a)

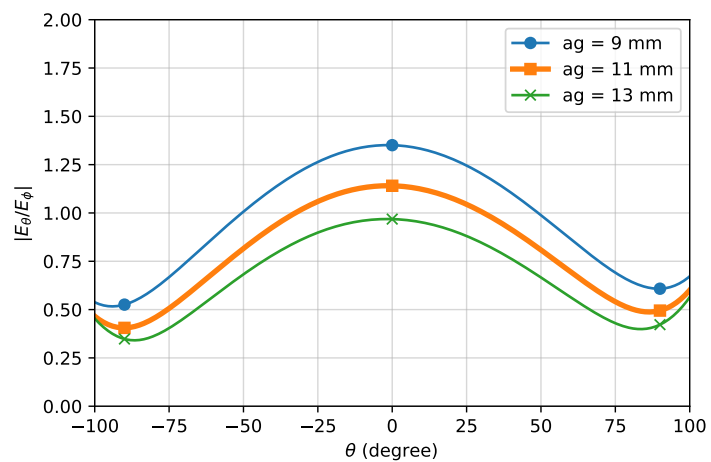

(c)

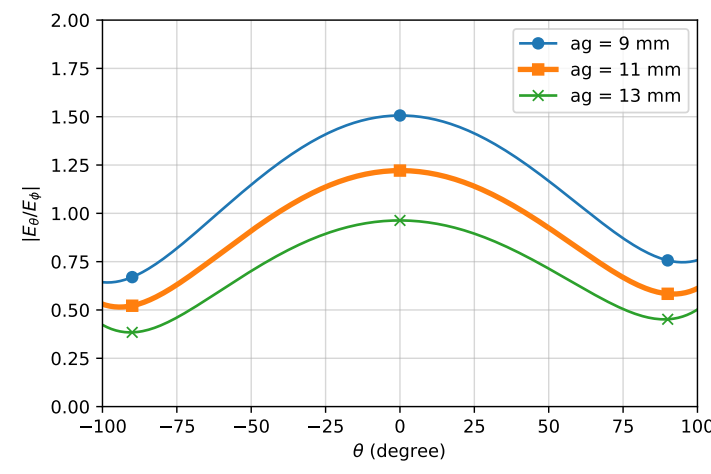

(b)

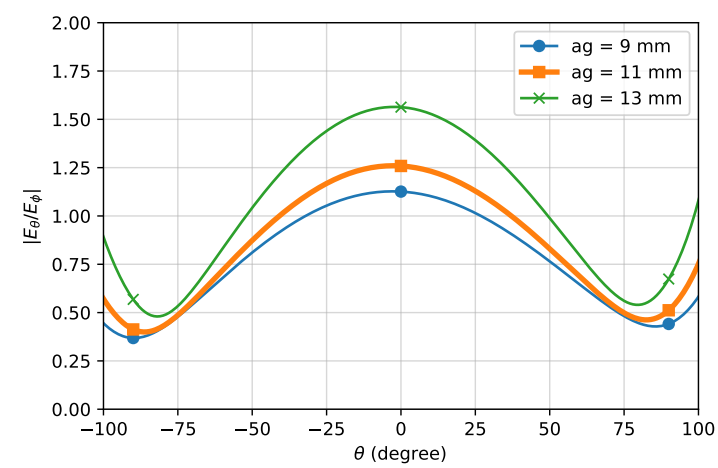

(d)

Figure 9. $E_{\theta}$ to $E_{\phi}$ ratio for the cut plane $\phi=0$ deg. (a) $f=2.0 \mathrm{GHz}$; (b) $f=2.1 \mathrm{GHz}$; (c) $f=2.2 \mathrm{GHz}$ and (d) $f=2.3 \mathrm{GHz}$.

In Figure 10 we show the phase difference between the two linear polarization $E_{\theta}$ and $E_{\phi}$, for the cut plane $\phi=0 \mathrm{deg}$. The on-axis $(\theta=0)$ phase difference, $\angle E_{\theta}-\angle E_{\phi}$, is differently affected at various frequencies as the air gap is increased. The required phase difference for CP is $90 \mathrm{deg}$. 
Figure 10 clearly shows that the phase difference $\angle E_{\theta}-\angle E_{\phi}$ has a strong dependence on the air gap. This effect is larger at higher frequencies.

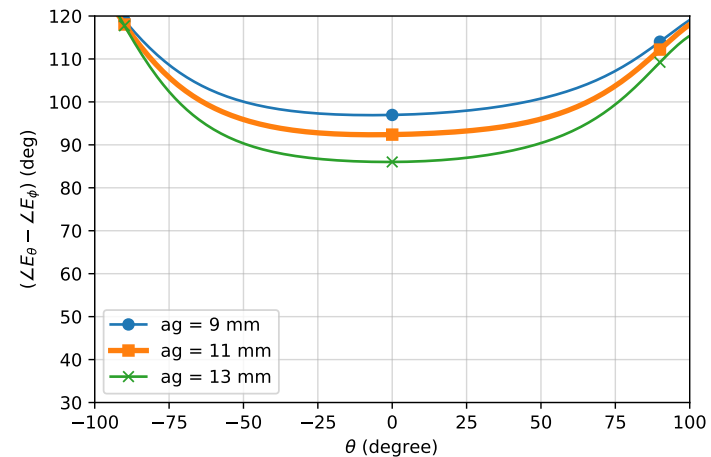

(a)

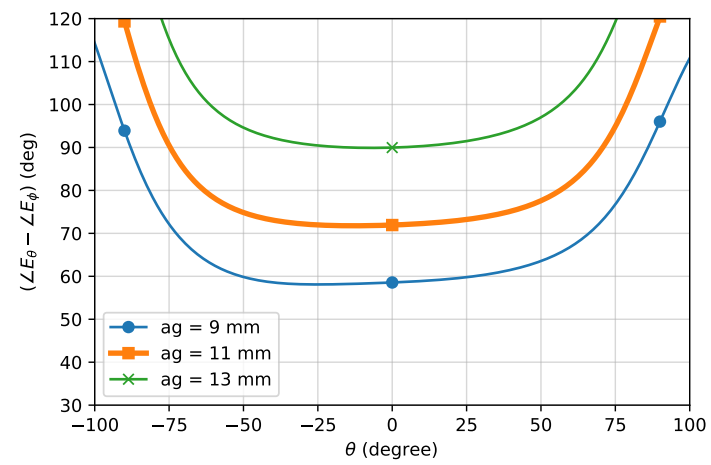

(c)

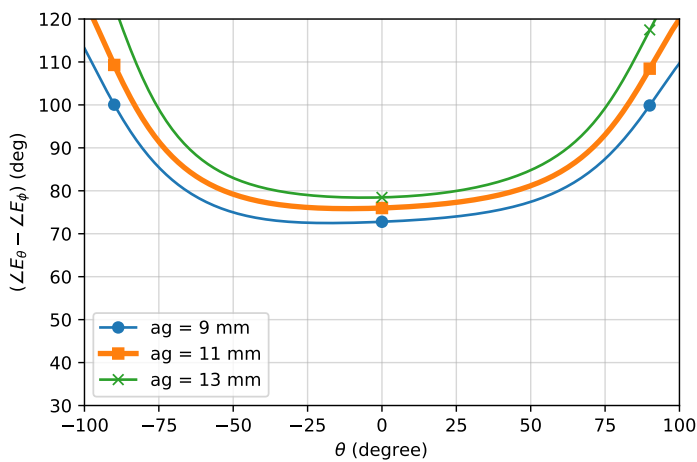

(b)

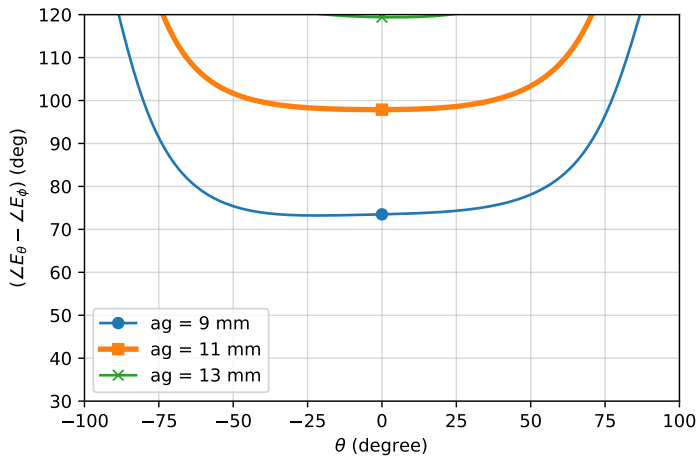

(d)

Figure 10. $\angle E_{\theta}-\angle E_{\phi}$ for the cut plane $\phi=0$ deg. (a) $f=2.0 \mathrm{GHz}$; (b) $f=2.1 \mathrm{GHz}$; (c) $f=2.2 \mathrm{GHz}$ and (d) $f=2.3 \mathrm{GHz}$.

In Table 2 we summarize the results of the parametric study on the air gap for the particular direction $\theta=0$.

Table 2. Linearly polarized component in the direction $\theta=0$.

\begin{tabular}{ccccc}
\hline & & $\mathbf{a g}=\mathbf{9}(\mathbf{m m})$ & $\mathbf{a g}=\mathbf{1 1}(\mathbf{m m})$ & $\mathbf{a g}=\mathbf{1 3}(\mathbf{m m})$ \\
\hline$f=2.0(\mathrm{GHz})$ & $\left(\left|E_{x}\right| /\left|E_{y}\right|\right)_{\theta=0}$ & 1.008 & 1.209 & 1.396 \\
& $\left(\angle E_{x}-\angle E_{y}\right)_{\theta=0}$ & 96.96 & 92.38 & 86.02 \\
\hline$f=2.1(\mathrm{GHz})$ & $\left(\left|E_{x}\right| /\left|E_{y}\right|\right)_{\theta=0}$ & 1.506 & 1.221 & 0.963 \\
& $\left(\angle E_{x}-\angle E_{y}\right)_{\theta=0}$ & 72.78 & 75.99 & 78.46 \\
\hline$f=2.2(\mathrm{GHz})$ & $\left(\left|E_{x}\right| /\left|E_{y}\right|\right)_{\theta=0}$ & 1.351 & 1.141 & 0.968 \\
& $\left(\angle E_{x}-\angle E_{y}\right)_{\theta=0}$ & 58.56 & 71.94 & 89.97 \\
\hline$f=2.3(\mathrm{GHz})$ & $\left(\left|E_{x}\right| /\left|E_{y}\right|\right)_{\theta=0}$ & 1.126 & 1.259 & 1.563 \\
& $\left(\angle E_{x}-\angle E_{y}\right)_{\theta=0}$ & 73.49 & 97.87 & 119.39 \\
\hline
\end{tabular}

We discussed Figures 9 and 10 for $\theta=0$ and in Table 2 we summarized only the case $\theta=0$. For the application at hand it is required a good CP up to $\theta=40 \mathrm{deg}$. The same figures can be used to control CP at several $\theta$ angles. With reference to Figure 9 we can observe that $\left|E_{\theta}\right| /\left|E_{\phi}\right|$ has been optimized to be close to 1 on "average" over a large $\theta$ interval. As it is well known the $E_{\theta}$ components drops at grating angles as it is apparent from the same two plots. This has been compensated here 
by choosing $\left|E_{\theta}\right| /\left|E_{\phi}\right|>1$ at $\theta=0$ but it can be corrected in several other manners, a well-known method is the use of a so-called "monopole fence", positioned around the patch element $[7,19]$.

The final set of parameters chosen for the antenna realization have been selected considering also other constraint such as the influence of the air gap on the impedance bandwidth, this study is shown in Figure 11.

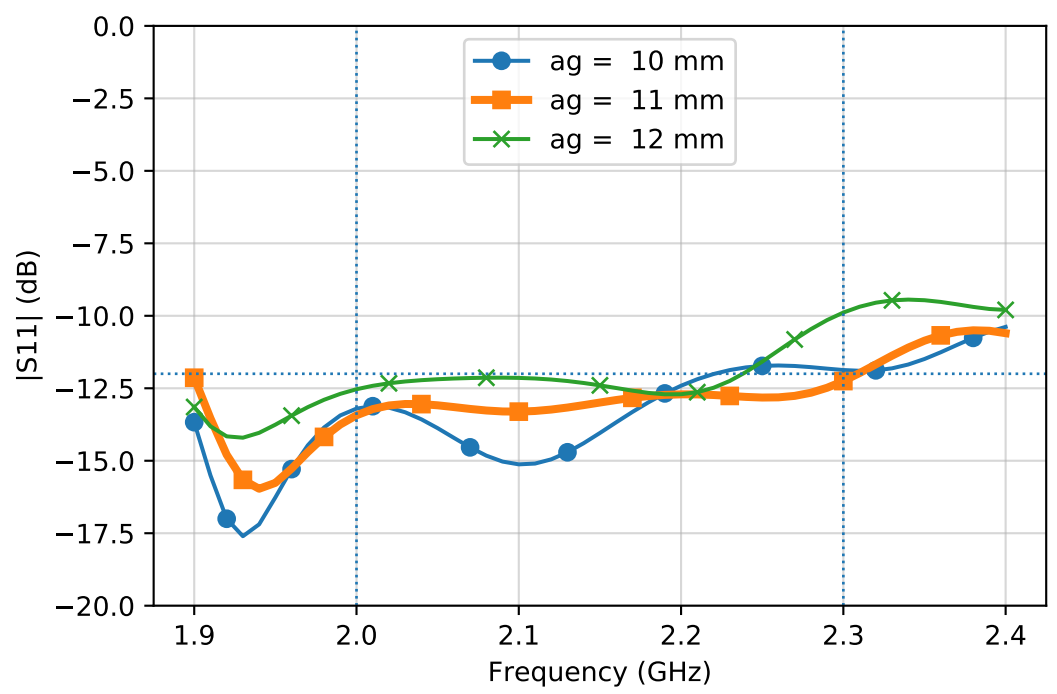

Figure 11. $\left|S_{11}\right|$ as a function of the air gap. The presence of the radome is included in this study. Variations in this parametric study are $9 \mathrm{~mm}, 11 \mathrm{~mm}$, and $13 \mathrm{~mm}$. The actual air gap of the realized antenna is $11.17 \mathrm{~mm}$ and is not shown in this parametric study since the curve is very close to the $11 \mathrm{~mm}$ one.

\section{Radome Geometry}

To guarantee protection from the external environment, a radome (Figure 2 in dashed blue) made of Delrin POM with $\epsilon_{r}=3.8$ and $\tan \delta=0.024$ has been designed and tested. Since the presence of the dielectric radome has an impact on the antenna performance the antenna optimization and fine-tuning have been carried out considering the presence of the radome. The geometry of the proposed antenna radome is shown in Figure 12.

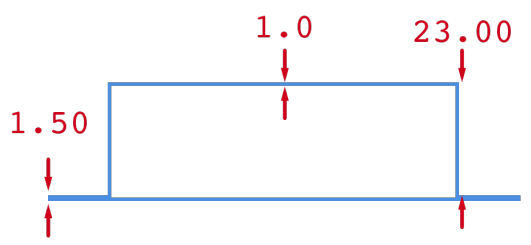

(a)

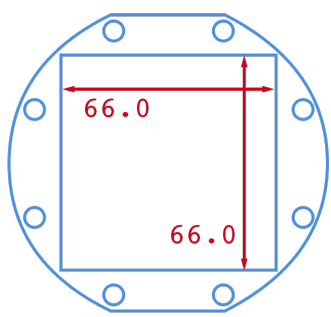

(b)

Figure 12. Side view (a) and top view (b) of the radome made of Delrin POM. Dimensions are shown in $\mathrm{mm}$.

The thickness of the square box is $1 \mathrm{~mm}$ and the total height is $23 \mathrm{~mm}$. On the circular base, with thickness of $1.5 \mathrm{~mm}$, eight circular holes are drilled to place the antenna on the platform.

The parametric study of the previous section, the optimization and the antenna characterization has been carried out considering the presence of the radome. The impact of the radome on the radiation pattern at $f=2.1 \mathrm{GHz}$ can be observed in Figure 13. 


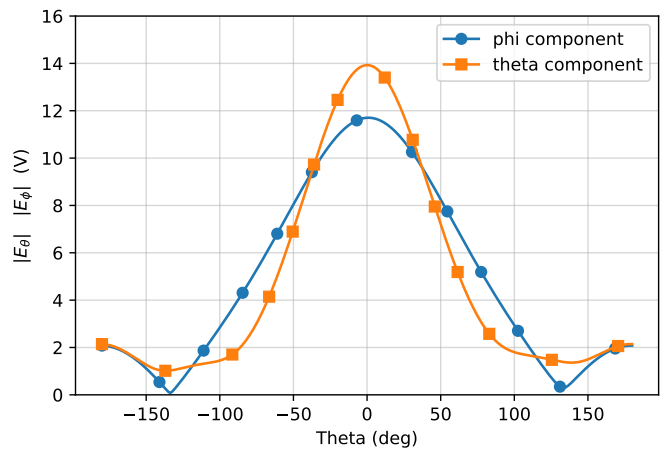

(a)

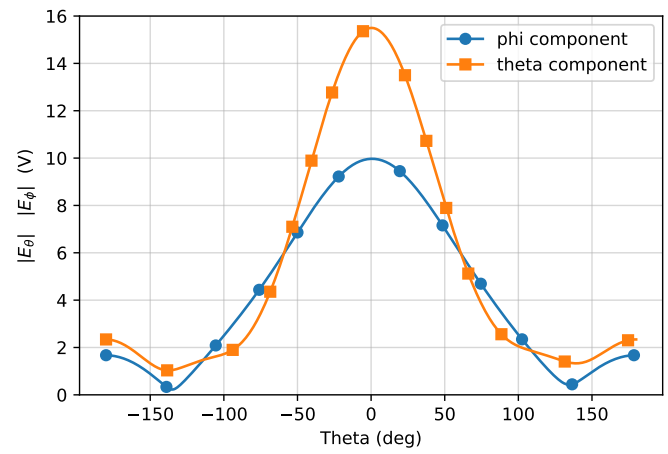

(b)

Figure 13. $E_{\theta}$ and $E_{\phi}$ components (a) with radome and (b) without radome, cut $\phi=0$ at $f=2.15 \mathrm{GHz}$.

In presence of the radome the two orthogonal modes/polarization $E_{\theta}$ and $E_{\phi}$ (that are tuned to oscillate in-phase quadrature) have almost the same amplitude $E_{\theta} / E_{\phi}=13.93 / 11.70=1.19$, due to the aforementioned optimization process. In absence of the radome, this ratio is slightly deteriorated $E_{\theta} / E_{\phi}=15.50 / 9.97=1.55$ as well as the axial ratio that reaches the value of $4.45 \mathrm{~dB}$ at $2.15 \mathrm{GHz}$.

\section{Results}

In the proposed work a total of four antennas (two RHCP and two LHCP) have been optimized to cover a frequency band from $2 \mathrm{GHz}$ to $2.3 \mathrm{GHz}$. To fully validate the antennas for space application, mechanical and thermal tests have been performed but are omitted in this paper for the sake of brevity. The manufactured prototype and the radome are shown in Figure 14.

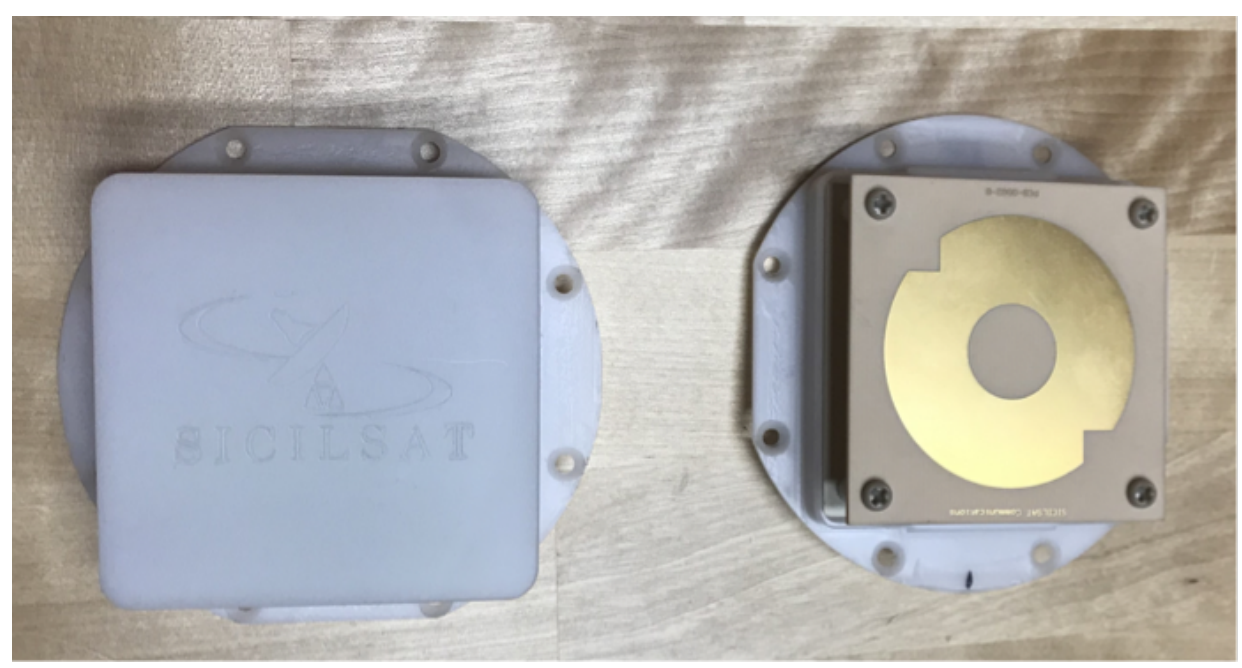

Figure 14. Manufactured prototype and radome.

Simulation and measurement of the return loss are shown in Figure 15. 


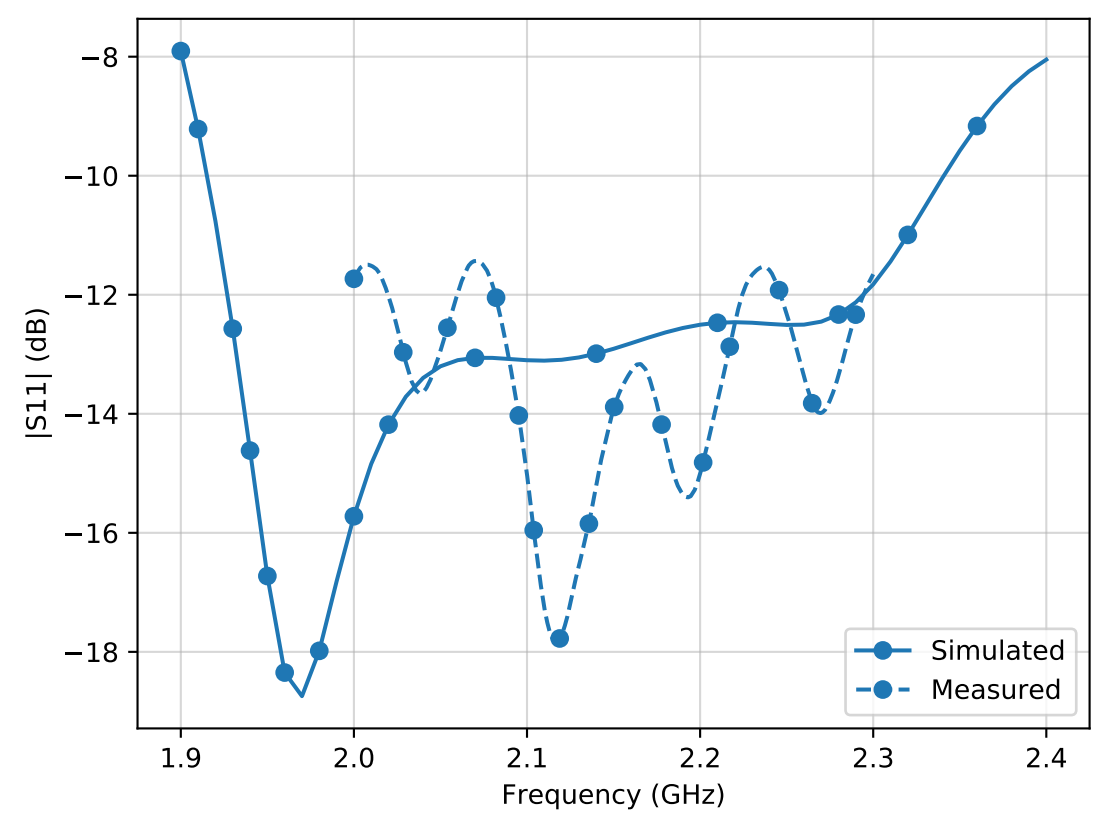

Figure 15. Simulated and measured $\left|S_{11}\right|$ for the proposed antenna.

The TT\&C frequency bandwidth where a $\left|S_{11}\right|<-10 \mathrm{~dB}$ ranges from $f_{L}=1.92 \mathrm{GHz}$ to $f_{H}=$ $2.34 \mathrm{GHz}$ which correspond to a bandwidth of $19.7 \%$. The antenna has been optimized to have an AR lower than $3.2 \mathrm{~dB}$ over the TT\&C bandwidth. The simulated and measured results for the AR and realized gain are presented in Figure 16. Simulations agree well with measurements.

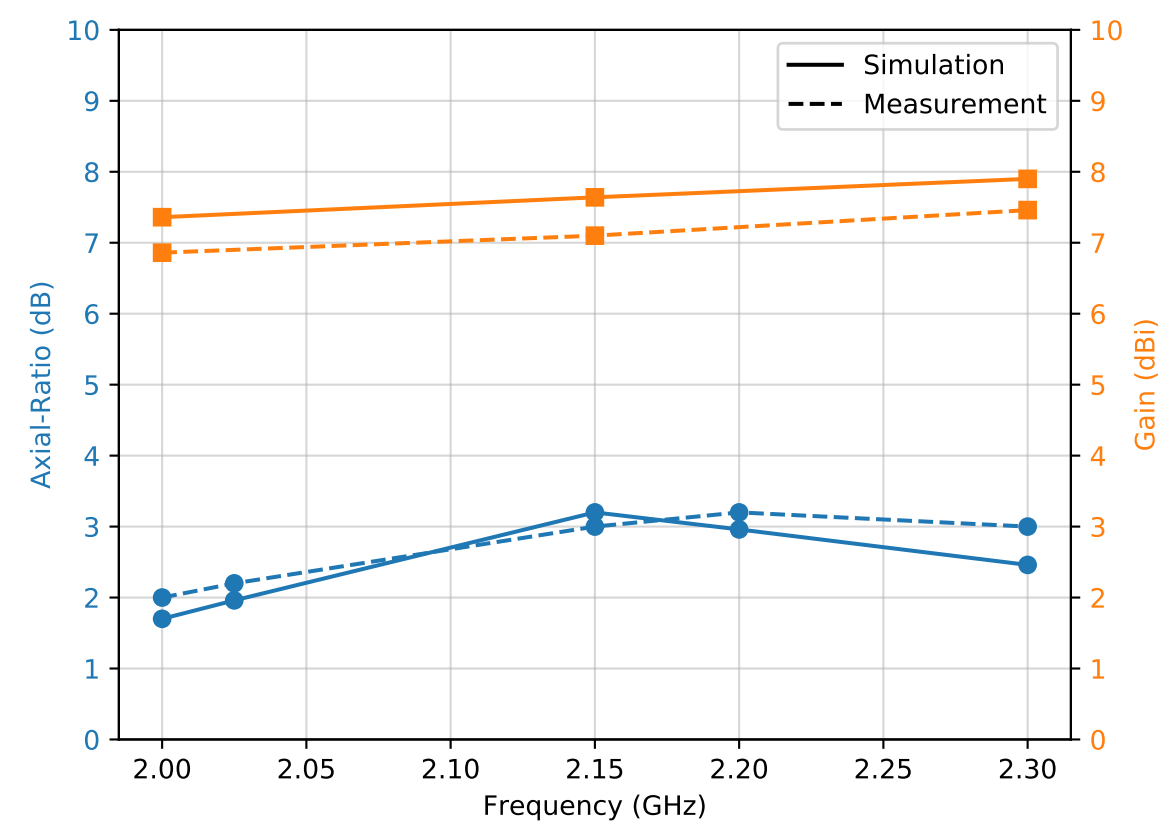

Figure 16. Simulated and measured axial ratio and realized gain for the proposed antenna. 
Figure 17 shows simulated and measured radiation patterns at different frequencies.

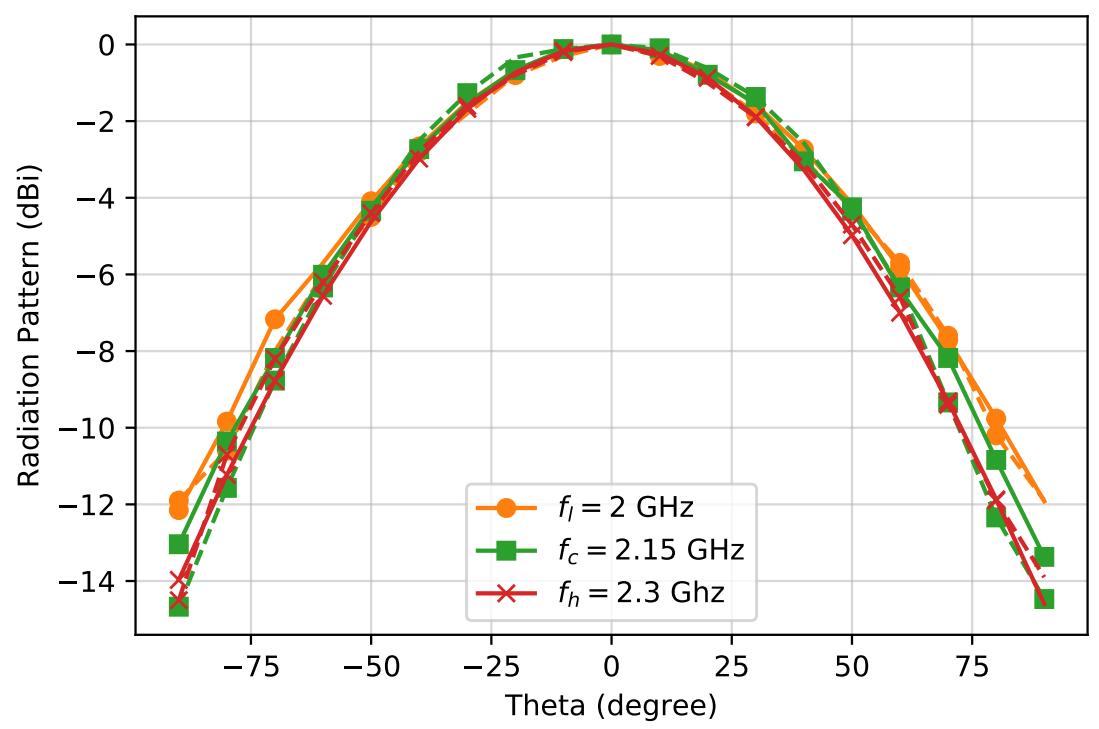

Figure 17. Simulated (solid line) and measured (dashed line) radiation patterns of the proposed antenna from $f_{l}=2 \mathrm{GHz}, f_{c}=2.15 \mathrm{GHz}$, and $f_{h}=2.3 \mathrm{GHz}$.

\section{Conclusions}

In this paper, a suspended patch antenna has been developed and optimized. The measured radiation pattern, axial ratio, and gain are in good agreement with the simulated ones and validate the electromagnetic design. The mechanical and thermal tests confirm that all the material used substrate, screws, as well as design criteria, are appropriate to get a space-qualified design. To realize a TT\&C system, four of these antennas (two RHCP and two LHCP) have been employed. In this way, a quasi-isotropic radiation pattern is obtained. It is worth pointing out that the chosen antenna design if fully compliant with the system requirements which are a $-3 \mathrm{~dB}$ beamwidth wider than $40^{\circ}$, a maximum gain higher than $6 \mathrm{dBi}$ and an axial ratio below $4 \mathrm{~dB}$ in the whole $2.0-2.3 \mathrm{GHz}$ operating band. Moreover, the proposed antenna exhibits a wide impedance bandwidth of $19.7 \%$.

Author Contributions: P.S. and C.S. defined the specific application requirements and selected the particular antenna type and geometry. G.S., L.D.D. and P.L. addressed conceptualization and methodology for the study, development, and analysis of circularly polarized antenna. P.S. and C.S. carried out the antenna optimization, and experimental characterizations. Writing-original draft preparation, P.S.; writing-review and editing, P.S, G.S., L.D.D. and P.L. The research was carried in the framework of an ongoing collaboration between the University of Palermo and the University of Catania, that more recently is also involving Aalborg University and Sicilsat Communications s.r.l, for the development of compact, low cost, circularly polarized antennas for telecommunications and wireless power transfer and harvesting.

Funding: This research was partially funded by the University of Catania with the project "Systems and technologies for 5G applications" under the program "Piano per la ricerca 2016-2018 - Linea di intervento 2".

Acknowledgments: The authors would like to thank Sicilsat Communications s.r.l. for providing technology and resources for the fabrication of the tested antenna.

Conflicts of Interest: The authors declare no conflict of interest. 


\section{Abbreviations}

The following abbreviations are used in this manuscript:

TT\&C Telemetry, Tracking and Command

CP Circularly Polarized

AR Axial Ratio

RHCP Right Hand Circular Polarized

LHCP Left Hand Circular Polarized

\section{References}

1. Tatomirescu, A.; Pedersen, G. Compact S Band Antenna for CubeSat. In Proceedings of the 2018 International Conference on Communications (COMM), Bucharest, Romania, 14-16 June 2018; pp. 231-234. [CrossRef]

2. Kramer, H.J.; Cracknell, A.P. An overview of small satellites in remote sensing. Int. J. Remote. Sens. 2008, 29, 4285-4337. [CrossRef]

3. Nascetti, A.; Pittella, E.; Teofilatto, P.; Pisa, S. High-Gain S-band Patch Antenna System for Earth-Observation CubeSat Satellites. IEEE Antennas Wirel. Propag. Lett. 2015, 14, 434-437. [CrossRef]

4. ITU. Radio Regulations. Available online: http://handle.itu.int/11.1002/pub/8063030b-en (accessed on 15 May 2019).

5. Ferrero, F.; Luxey, C.; Jacquemod, G.; Staraj, R. Dual-band circularly polarized microstrip antenna for satellite applications. IEEE Antennas Wirel. Propag. Lett. 2005, 4, 13-15. [CrossRef]

6. Choi, E.; Lee, J.W.; Lee, T. Modified S-Band Satellite Antenna With Isoflux Pattern and Circularly Polarized Wide Beamwidth. IEEE Antennas Wirel. Propag. Lett. 2013, 12, 1319-1322. [CrossRef]

7. Choi, E.; Lee, J.W.; Lee, T.; Lee, W. Circularly Polarized S-Band Satellite Antenna With Parasitic Elements and Its Arrays. IEEE Antennas Wirel. Propag. Lett. 2014, 13, 1689-1692. [CrossRef]

8. Veljovic, M.J.; Skrivervik, A.K. Aperture-Coupled Low-Profile Wideband Patch Antennas for CubeSat. IEEE Trans. Antennas Propag. 2019, 67, 3439-3444. [CrossRef]

9. Hu, W.; Wen, G.; Inserra, D.; Huang, Y.; Li, J.; Chen, Z.D. A Circularly Polarized Antenna Array with Gain Enhancement for Long-Range UHF RFID Systems. Electronics 2019, 8, 400. [CrossRef]

10. Samsuzzaman, M.; Islam, M.T.; Faruque, M.R.I. Circularly polarized dual frequency patch antenna for TTC applications. In Proceedings of the 2013 Third World Congress on Information and Communication Technologies (WICT 2013), Hanoi, Vietnam, 15-18 December 2013; pp. 258-261. [CrossRef]

11. Padilla, J.; Rosati, G.; Ivanov, A.; Bongard, F.; Vaccaro, S.; Mosig, J. Multi-functional miniaturized slot antenna system for small satellites. In Proceedings of the 5th European Conference on Antennas and Propagation (EUCAP), Rome, Italy, 11-15 April 2011; pp. 2170-2174.

12. Leonardi, O.; Pavone, M.G.; Sorbello, G.; Morabito, A.F.; Isernia, T. Compact single-layer circularly polarized antenna for short-range communication systems. Microw. Opt. Technol. Lett. 2014, 56, 1843-1846. [CrossRef]

13. Zaid, J.; Abdulhadi, A.; Kesavan, A.; Belaizi, Y.; Denidni, T. Multiport circular polarized RFID-tag antenna for UHF sensor applications. Sensors 2017, 17, 1576. [CrossRef] [PubMed]

14. Sitompul, P.P.; Sumantyo, S.; Tetuko, J.; Kurniawan, F.; Nasucha, M. Axial Ratio and Gain Enhancement of a Circular-Ring Slot Antenna Using a Pair of Asymmetrical Rectangular Slots and a Parasitic Patch for a Radio Beacon on a Nanosatellite. Aerospace 2019, 6, 39. [CrossRef]

15. Leonardi, O.; Pavone, M.; Cadili, T.; Sorbello, G.; Isernia, T. Monolithic patch antenna for dedicated short-range communications. Electron. Lett. 2013, 49, 85-86. [CrossRef]

16. Di Carlo, C.; Di Donato, L.; Mauro, G.; La Rosa, R.; Livreri, P.; Sorbello, G. A circularly polarized wideband high gain patch antenna for wireless power transfer. Microw. Opt. Technol. Lett. 2018, 60, 620-625. [CrossRef]

17. Kokotoff, D.M.; Aberle, J.T.; Waterhouse, R.B. Rigorous analysis of probe-fed printed annular ring antennas. IEEE Trans. Antennas Propag. 1999, 47, 384-388. [CrossRef]

18. Someda, C.G. Electromagnetic Waves; CRC Press: Boca Raton, FL, USA, 2006. 
19. Mauro, G.S.; Torrisi, G.; Mariano, P.D.; Squadrito, C.; Emanuele, S.; Donato, L.D.; Sorbello, G. Wide Bandwidth Dual Port, Dual Sense Circular Polarization Antenna for Satellite Applications. In Proceedings of the ICEAA 2019 International Conference on Electromagnetics in Advanced Applications, IEEE-APS Topical Conference on Antennas and Propagation in Wireless Communications, Granada, Spain, 9-13 September 2019. 